\title{
Enhanced recovery after surgery pathways in gynecologic surgery: great strides already, but more still to come
}

William J Fawcett

\section{Correspondence to} Professor William J Fawcett, Anaesthesia and Pain Medicine, Royal Surrey County Hospital NHS Foundation Trust, Guildford GU2 7XX, UK; wfawcett@nhs. net

Received 26 February 2019 Accepted 28 February 2019

Check for updates

(C) IGCS and ESGO 2019. № commercial re-use. See rights and permissions. Published by BMJ.

To cite: Fawcett WJ. Int $J$ Gynecol Cancer 2019;29:649650.
Enhanced recovery after surgery (ERAS) has revolutionized the peri-operative care of patients undergoing major elective surgery over the last two decades. Its principle pioneer, Henrik Kehlet, a Danish surgeon, established a philosophy to provide a multidisciplinary, multimodal approach to patient care pathways to minimize peri-operative physiologic dysfunction and the surgical stress response, resulting in a swifter return to normal function.

Initially there was a focus in colorectal surgery but more recently the concept has been embraced by many other specialties including orthopedics, esophagogastric, hepatobiliary, urology, gynecology, bariatric, head and neck, and thoracic and obstetrics surgeries. ${ }^{1}$ of interest and relevance here is that gynecologic oncology surgery is major, complex surgery that interfaces with 'benign' gynecology, colorectal, and urology so it occupies a central role within ERAS guidelines.

An early driver for ERAS was to reduce hospital length of stay. One reason for this was that this is a universally measured metric and reductions in hospital stay have benefits for patients, clinicians, and managers ${ }^{2}$ provided that re-admissions are not increased as a result. However, there are now data that not only support reduced re-admissions, but demonstrate that there is a wealth of other benefits for ERAS patients, including reduced complications, improved outcome, and reduced costs. ${ }^{3}$ The link between complications and outcome is well known, ${ }^{4}$ and as ERAS becomes more established, we are now beginning to see data showing that longer-term outcomes are improved, for example in patients with colorectal cancer on ERAS programs. ${ }^{5}$ These findings may be partly due to reduced early complications, but in addition there may be effects of long-term preservation of immune function and because patients are fit enough to tolerate other adjuvant treatments more quickly such as chemotherapy and radiotherapy. ${ }^{2}$

In this month's journal, the guidelines for gynecologic surgery are revised and updated ${ }^{6}$ and provide an important time to reflect on what is new. As the ERAS evidence base grows, so the grade of evidence may change as well as new evidence emerging to further improve patient care. The latter includes the emerging area of prehabilitation, as well as pelvic exenteration and hyperthermic intra-peritoneal chemotherapy. It is good to see focus on the post-operative period such as discharge pathways and patient-reported outcomes. In addition, the post-operative period is viewed as probably the most difficult area to implement, yet when successfully undertaken is most strongly associated with good outcomes, such as early removal of urinary catheters, ambulation, and oral feeding. ${ }^{78}$

While our knowledge of ERAS will continue to move forward with well-conducted standardized studies, ${ }^{9}$ and with good pathway adherence required to derive the maximal benefits, it must also be borne in mind that evidence for some of the pathways is now less compelling. ${ }^{10}{ }^{11}$ Crucially, there is much that individual institutions can achieve, in particular by collecting good-quality audit data and comparing this with recognized centers of excellence. ${ }^{8}$ Moreover, it is not enough to know what to do in theory; it is the continued practical implementation of ERAS that is essential. Finally, as the benefits of ERAS are recognized, the concepts need to be taught more widely, not only at postgraduate level, but to undergraduates too.

We have come a long way in the last 20 years from the concept popularized by Henrik Kehlet, but safe, quality care is always the goal - as Kehlet himself says: "First do it better, then do it quicker".

Funding The author has not declared a specific grant for this research from any funding agency in the public, commercial or not-for-profit sectors.

Competing interests Executive Committee Member of ERAS ${ }^{\circ}$ Society.

Patient consent for publication Not required.

Provenance and peer review Commissioned; internally peer reviewed.

\section{REFERENCES}

1. ERAS $®$ Society. Available: http://erassociety.org/ guidelines/list-of-guidelines/ 


\section{Editorial}

2. Fawcett WJ, Mythen MG, Scott MJP. Enhanced recovery: more than just reducing length of stay? Br J Anaesth 2012;109:671-4.

3. Ljungqvist $\mathrm{O}$, Scott $\mathrm{M}$, Fearon $\mathrm{KC}$. Enhanced recovery after surgery: a review. JAMA Surg 2017;152:292-8.

4. Khuri SF, Henderson WG, DePalma RG, et al. Determinants of long-term survival after major surgery and the adverse effect of postoperative complications. Ann Surg 2005;242:326-43.

5. Gustafsson UO, Oppelstrup $\mathrm{H}$, Thorell A, et al. Adherence to the ERAS protocol is associated with 5-year survival after colorectal cancer surgery: a retrospective cohort study. World J Surg 2016;40:1741-7.

6. Nelson G, Bakkum-Gamez J, Kalogera E, et al. Guidelines for perioperative care in Gynecologic/Oncology: Enhanced recovery

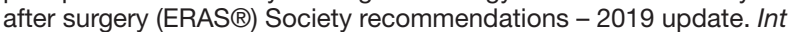
J Gynecol Cancer. 2019, In Press.

7. Aarts M-A, Rotstein OD, Pearsall EA, et al. Postoperative ERAS interventions have the greatest impact on optimal recovery: experience with implementation of ERAS across multiple hospitals. Ann Surg 2018;267:992-7.

8. Kehlet H. ERAS Implementation-Time to move forward. Ann Surg 2018;267:998-9.

9. Elias $\mathrm{KM}$, Stone $\mathrm{AB}$, McGinigle $\mathrm{K}$, et al. The reporting on eras compliance, outcomes, and elements research (recover) checklist: a

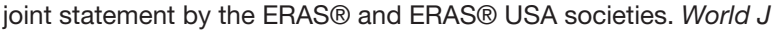
Surg 2018;16:1-8.

10. Amer MA, Smith MD, Herbison GP, et al. Network meta-analysis of the effect of preoperative carbohydrate loading on recovery after elective surgery. Br J Surg 2017;104:187-97.

11. Rollins KE, Lobo DN. Intraoperative goal-directed fluid therapy in elective major abdominal surgery: a meta-analysis of randomized controlled trials. Ann Surg 2016;263:465-76. 